COLORECTAL CANCER

\title{
Incomplete screening flexible sigmoidoscopy associated with female sex, age, and increased risk of colorectal cancer
}

\author{
V P Doria-Rose, P A Newcomb, T R Levin
}

See end of article for authors' affiliations

Correspondence to Dr V P Doria-Rose, Fred Hutchinson Cancer Research Center, 1100 Fairview Ave North, M4B402, PO Box 19024,

Seattle, Washington 98109-1024, USA; pdoriaro@fhcrc.org

Revised version received 14 April 2005

Accepted for publication 26 April 2005

Published online first

4 May 2005
Background: Several previous studies have found that females and older individuals are at greater risk of having incomplete flexible sigmoidoscopy. However, no prior study has reported the subsequent risk of colorectal cancer (CRC) following incomplete sigmoidoscopy.

Methods: Using data from 55791 individuals screened as part of the Colon Cancer Prevention (CoCaP) programme of Kaiser Permanente of Northern California, we evaluated the likelihood of having an inadequate $(<40 \mathrm{~cm})$ examination by age and sex, and estimated the risk of distal CRC according to depth of sigmoidoscope insertion at the baseline screening examination. Multivariate estimation of risks was performed using Poisson regression.

Results: Older individuals were at a much greater risk of having an inadequate examination (relative risk (RR) for age 80+ years compared with 50-59 years 2.6 (95\% confidence interval (CI) 2.3-3.0)), as were females (RR 2.3 (95\% Cl 2.2-2.5)); these associations were attenuated but remained strong if Poisson models were further adjusted for examination limitations (pain, stool, and angulation). There was an approximate threefold increase in the risk of distal CRC if the baseline sigmoidoscopy did not reach a depth of at least $40 \mathrm{~cm}$; a smaller increase in risk was observed for examinations that reached $40-59 \mathrm{~cm}$. Conclusions: Older individuals and women are at an increased risk of having inadequate sigmoidoscopy. Because inadequate sigmoidoscopy results in an increased risk of subsequent CRC, physicians should consider steps to maximise the depth of insertion of the sigmoidoscope or, failing this, should consider an alternative screening test.
S creening by flexible sigmoidoscopy is associated with both decreased incidence of ${ }^{12}$ and decreased mortality from $^{3}{ }^{4}$ colorectal cancer (CRC). However, the efficacy of sigmoidoscopy is dependent on adequate visualisation of the colorectal mucosa so that colorectal polyps can be identified and removed before they progress to invasive cancer. Therefore, an inadequate examination (that is, one that has a limited depth of insertion of the sigmoidoscope or is hindered by poor bowel preparation or patient discomfort) may lead to a reduction in efficacy.

Previous studies have found that women and older individuals are more likely to have an inadequate sigmoidoscopy, usually defined as an examination reaching less than $50 \mathrm{~cm}$ depth of insertion (for a $60 \mathrm{~cm}$ sigmoidoscope), or limited by poor bowel preparation, pain, and/or angulation. ${ }^{5-9}$ The choice of $50 \mathrm{~cm}$ as a cutoff is somewhat arbitrary, however, and to our knowledge no prior study has correlated the definition of "inadequate" or "incomplete" sigmoidoscopy with the future risk of distal CRC (that is, cancers within reach of the sigmoidoscope). The purpose of this study was to evaluate cross sectionally risk factors for inadequate sigmoidoscopy at baseline screening, and further to conduct a cohort analysis of the association of sigmoidoscopy depth of insertion and examination limitations with distal CRC diagnosis within an approximate five year period postsigmoidoscopy, using data from a large health maintenance organisation.

\section{METHODS}

\section{Subjects and data sources}

The study included men and women aged 50 years and older who were considered not to be at high risk of developing CRC and who participated in Kaiser Permanente of Northern
California's (KP) Colon Cancer Prevention (CoCaP) programme. ${ }^{10} \mathrm{KP}$ is a large prepaid health plan of approximately 2.75 million members in the San Francisco Bay area of California; the CoCaP programme offers a screening flexible sigmoidoscopy to all KP members aged 50 years and older once every 10 years.

In 1994 and 1995, reports from all sigmoidoscopies performed in KP facilities were entered into a CoCaP computerised database. These reports included patient reported medical history and indications for examination (screening versus symptoms), plus endoscopist recorded indications for examination and results, including depth of insertion, limitations of the examination (due to spasm/pain, stool, or angulation), and number and depth of any polyps identified. Histological findings from any removed or biopsied polyps were linked back to the sigmoidoscopy data form. Additional demographic data (age and sex) and provider data (gastroenterologist, non-gastroenterologist physician, or nurse) were obtained from other KP databases, and incident cases of CRC to 31 December 2000 were identified using the KP Tumor Registry.

The study included screening sigmoidoscopies (as determined by both patient and endoscopist reported indication for examination) performed in 1994 and 1995 among CoCaP participants. Either patient or endoscopist report that the examination was being done as a result of symptoms resulted in exclusion. If a patient had more than one sigmoidoscopy during this time period, only the first was considered. Patients at high risk of developing CRC were excluded, based on the presence of inflammatory bowel disease, prior

Abbreviations: $\mathrm{CoCaP}$, colon cancer prevention; $\mathrm{CRC}$, colorectal cancer; KP, Kaiser Permanente of Northern California; RR, relative risk 


\begin{tabular}{|c|c|}
\hline Characteristic & № (\%) \\
\hline \multicolumn{2}{|l|}{ Age (y) } \\
\hline $50-59$ & $26141(46.9)$ \\
\hline $60-69$ & $20452(36.7)$ \\
\hline $70-79$ & $8381(15.0)$ \\
\hline $80+$ & $817(1.5)$ \\
\hline \multicolumn{2}{|l|}{ Sex } \\
\hline Male & $29537(52.9)$ \\
\hline Female & $26248(47.1)$ \\
\hline \multicolumn{2}{|l|}{ Endoscopist specially } \\
\hline Gastroenterologist & $15199(27.2)$ \\
\hline Non-gastroenterologist MD & $29036(52.0)$ \\
\hline Nurse & 8111 (14.5) \\
\hline Unknown & $3445(6.2)$ \\
\hline \multicolumn{2}{|l|}{ Family history of CRC* } \\
\hline No & 51486 (92.3) \\
\hline Yes & $4305(7.7)$ \\
\hline \multicolumn{2}{|l|}{ History of prior sigmoidoscopy } \\
\hline No & $41836(76.1)$ \\
\hline Yes & 13121 (23.9) \\
\hline \multicolumn{2}{|c|}{ Depth of sigmoidoscope insertion $(\mathrm{cm})$} \\
\hline$<30$ & $1733(3.1)$ \\
\hline $30-39$ & $3897(7.0)$ \\
\hline $40-49$ & $6061(10.9)$ \\
\hline $50-59$ & $8522(15.3)$ \\
\hline $60+$ & $35578(63.8)$ \\
\hline \multicolumn{2}{|l|}{ Most advanced finding } \\
\hline No polyps & $45863(82.2)$ \\
\hline Non-adenomatous polyp & $5035(9.0)$ \\
\hline Non-advanced adenoma & $3970(7.1)$ \\
\hline Advanced adenoma† & $923(1.7)$ \\
\hline \multicolumn{2}{|l|}{ Examination limited by } \\
\hline Spasm/pain & 9967 (17.9) \\
\hline Stool & $7959(14.3)$ \\
\hline Angulation & 8030 (14.4) \\
\hline
\end{tabular}

colorectal polyps or cancer, or history of CRC in more than one first degree relative or one first degree relative diagnosed at age 55 years or younger. ${ }^{11}$ Additionally, we excluded those who had CRC diagnosed at baseline, as identification of a lesion suspected of being cancer often results in termination of the examination prior to the maximal possible depth of insertion. Finally, we excluded those who had no indication of insertion depth recorded on the sigmoidoscopy report. This research was approved by the institutional review boards at Kaiser Permanente of Northern California (Oakland, California, USA) and at the Fred Hutchinson Cancer Research Center (Seattle, Washington, USA).

\section{Statistical analysis}

We chose to define "inadequate" sigmoidoscopy as those examinations that reached a depth of less than $40 \mathrm{~cm}$, based on the subsequent incidence rates of distal CRC according to sigmoidoscopy insertion depth (see below). The proportion of individuals who had inadequate sigmoidoscopy was calculated for groups defined by both age and sex, and 95\% confidence intervals (CI) for the proportions were calculated. Finally, we calculated the adjusted relative risk (RR) of an inadequate examination, and corresponding 95\% CI, using Poisson regression with the robust estimator of variance. These models allow for valid estimation of RR and CI when the outcome of interest is common (that is, when the odds

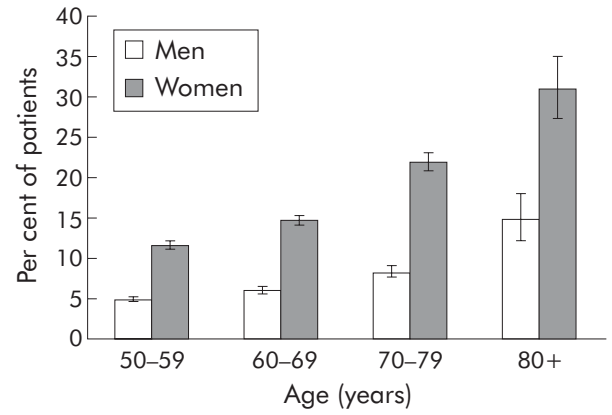

Figure 1 Proportion of men and women with inadequate sigmoidoscopy, by age. Error bars represent $95 \%$ confidence intervals for the proportion.

ratio is a poor approximation of the relative risk). ${ }^{12}$ All Poisson models were adjusted for age (50-59, 60-69, 70-79, and $80+$ years), sex, history of prior sigmoidoscopy, and family history of CRC.

Incidence rates of distal CRC (that is, cancers of the rectum and sigmoid colon, ICD-0-2 codes C18.7, C19.9, and C20.9) were calculated by categories of sigmoidoscopy depth of insertion and examination limitations. Rates were calculated by dividing the number of distal CRC cases by the total amount of person-time at risk. Study subjects were followed until 31 December 2000 or until the time of death, KP membership termination, or CRC diagnosis. Poisson regression was used to estimate the rate ratios, with $95 \% \mathrm{CI}$, for sigmoidoscopy depth of insertion and examination limitations. All Poisson models were adjusted using indicator variables for age (as parameterised above), sex, and family history of CRC. Analyses were conducted using the statistical software package STATA, version 8.0 (Stata Corporation, College Station, Texas, USA)

\section{RESULTS}

Results from 55791 sigmoidoscopies were included in this analysis. Mean age of study subjects was 61.7 years, and $47.1 \%$ of subjects were women. Just over one half of examinations were performed by non-gastroenterologist physicians. Less than $10 \%$ of patients had a history of CRC in one first degree relative diagnosed after the age of 55 years, and approximately $25 \%$ had undergone previous sigmoidoscopy. Almost two thirds of sigmoidoscopic examinations reached $60 \mathrm{~cm}$ or greater; examinations to greater than $60 \mathrm{~cm}$ (3\% of all examinations) largely occurred due to the use of a $65 \mathrm{~cm}$ sigmoidoscope in a small proportion of patients. Approximately $10 \%$ of sigmoidoscopies were to less than $40 \mathrm{~cm}$. Eighteen per cent of screenees had polyps identified at sigmoidoscopy; about $50 \%$ of those with polyps had adenomas, and less than $2 \%$ had an advanced adenoma detected (defined as a tubular adenoma, $\geqslant 1 \mathrm{~cm}$ in size, or with villous histology or severe dysplasia) (table 1).

The proportion of inadequate sigmoidoscopies increased dramatically with increasing age and female sex (fig 1), ranging from 5\% in men aged 50-59 years to $29 \%$ in women aged 80 years and older. These patterns were very similar if alternate definitions of "inadequate" were used. For men aged $50-59,12 \%$ had examinations to less than $50 \mathrm{~cm}$, and $25 \%$ of examinations did not reach $60 \mathrm{~cm}$ or more. In women aged 80 years and older, $47 \%$ and $65 \%$ of examinations did not reach 50 and $60 \mathrm{~cm}$, respectively.

In the multivariate Poisson model, there was a greater than twofold increase in the risk of inadequate sigmoidoscopy for both women and for those over age 80 years. There was no evidence of an interaction between sex and age. The risk was also increased when the provider was not a 
Table 2 Relative risk of inadequate sigmoidoscopy by patient and examination characteristics, with and without adjustment for examination limitations

\begin{tabular}{|c|c|c|c|c|c|}
\hline \multirow[b]{2}{*}{ Characteristic } & \multirow{2}{*}{$\begin{array}{l}\text { Proportion with } \\
\text { inadequate } \\
\text { sigmoidoscopy (\%) }\end{array}$} & \multicolumn{2}{|c|}{ Not limitations adjusted } & \multicolumn{2}{|c|}{ Limitations adjustec } \\
\hline & & $\mathrm{RR}^{*}$ & $95 \% \mathrm{Cl}$ & $\mathbf{R R}^{*}$ & $95 \% \mathrm{Cl}$ \\
\hline \multicolumn{6}{|l|}{ Age (y) } \\
\hline $50-59$ & 8.1 & 1.0 & Ref. & 1.0 & Ref. \\
\hline $60-69$ & 10.3 & 1.2 & $1.2-1.3$ & 1.2 & $1.1-1.2$ \\
\hline $70-79$ & 14.6 & 1.7 & $1.6-1.8$ & 1.5 & $1.4-1.6$ \\
\hline $80+$ & 22.0 & 2.6 & $2.3-3.0$ & 1.8 & $1.6-2.1$ \\
\hline \multicolumn{6}{|l|}{ Sex } \\
\hline Male & 6.1 & 1.0 & Ref. & 1.0 & Ref. \\
\hline Female & 14.6 & 2.3 & $2.2-2.5$ & 1.6 & $1.6-1.7$ \\
\hline \multicolumn{6}{|l|}{ Endoscopist specialty } \\
\hline Gastroenterologist & 7.8 & 1.0 & Ref. & 1.0 & Ref. \\
\hline Non-gastroenterologist MD & 10.0 & 1.3 & $1.2-1.4$ & 1.2 & $1.2-1.3$ \\
\hline Nurse & 7.9 & 1.0 & $0.9-1.0$ & 1.0 & $0.9-1.1$ \\
\hline Unknown & 26.5 & 3.3 & $3.0-3.5$ & 2.1 & $1.9-2.3$ \\
\hline \multicolumn{6}{|l|}{ Family history of CRC $\dagger$} \\
\hline No & 10.2 & 1.0 & Ref. & 1.0 & Ref. \\
\hline Yes & 9.4 & 0.9 & $0.8-1.0$ & 0.9 & $0.8-1.0$ \\
\hline \multicolumn{6}{|l|}{ History of prior sigmoidoscopy } \\
\hline No & 9.7 & 1.0 & Ref. & 1.0 & Ref. \\
\hline Yes & 11.2 & 1.1 & $1.0-1.2$ & 1.0 & $1.0-1.1$ \\
\hline \multicolumn{6}{|l|}{ Examination limited by } \\
\hline \multicolumn{6}{|l|}{ Spasm/pain } \\
\hline No & 5.6 & - & - & 1.0 & Ref. \\
\hline Yes & 30.8 & - & - & 3.1 & $2.9-3.3$ \\
\hline \multicolumn{6}{|l|}{ Stool } \\
\hline No & 8.6 & - & - & 1.0 & Ref. \\
\hline Yes & 18.8 & - & - & 2.9 & $2.7-3.0$ \\
\hline \multicolumn{6}{|l|}{ Angulation } \\
\hline No & 6.1 & - & - & 1.0 & Ref. \\
\hline Yes & 34.1 & - & - & 2.9 & $2.8-3.1$ \\
\hline
\end{tabular}

gastroenterologist, especially for those providers for whom specialty could not be determined from the sigmoidoscopy report; neither family history nor history of prior sigmoidoscopy was a strong risk factor for inadequate sigmoidoscopy. Further adjustment of the full Poisson model for sigmoidoscopy limitations resulted in attenuation of all relative risks; however, the associations with sex, age, and unknown specialty remained quite strong. Each individual limitation was independently associated with an approximate threefold increase in risk (table 2). These relationships were quite similar if the sample was restricted to those who had no polyps identified at sigmoidoscopy, or to those with no limitations noted on the sigmoidoscopy report.

When sigmoidoscopy limitations were considered as an outcome in Poisson models, increasing age was a risk factor for examinations limited by spasm/pain, angulation, and especially by stool. Female sex was a risk factor for examinations limited by pain or angulation, but not by stool (table 3).

Depth of sigmoidoscope insertion was associated with the discovery of polyps at baseline. While there was a tendency for polyp prevalence to increase with increasing depth of insertion, those with examinations to less than $30 \mathrm{~cm}$ had a higher prevalence of polyps than all but those with examinations to $60 \mathrm{~cm}$ or more. Polyps were identified in $17.7 \%$ of sigmoidoscopies with a depth of insertion less than $30 \mathrm{~cm}$, and $14.2 \%, 14.6 \%, 15.9 \%$, and $19.2 \%$ of examinations to $30-39,40-49,50-59$, and $60 \mathrm{~cm}$ or more, respectively. This increased prevalence among those with examinations to less than $30 \mathrm{~cm}$ was most pronounced for advanced adenomas; the corresponding advanced adenoma prevalences for increasing depth of insertion categories were $2.9 \%, 1.7 \%$,

Table 3 Relative risk of sigmoidoscopy limitations by patient age and sex

\begin{tabular}{|c|c|c|c|c|c|c|}
\hline \multirow[b]{3}{*}{ Characteristic } & \multicolumn{6}{|c|}{ Limitation } \\
\hline & \multicolumn{2}{|c|}{ Spasm/pain } & \multicolumn{2}{|l|}{ Stool } & \multicolumn{2}{|c|}{ Angulation } \\
\hline & $\mathbf{R R}^{*}$ & $95 \% \mathrm{Cl}$ & $\mathbf{R R}^{*}$ & $95 \% \mathrm{Cl}$ & $\mathbf{R R}^{*}$ & $95 \% \mathrm{Cl}$ \\
\hline \multicolumn{7}{|l|}{ Age (y) } \\
\hline $50-59$ & 1.0 & Ref. & 1.0 & Ref. & 1.0 & Ref. \\
\hline $60-69$ & 1.0 & $1.0-1.1$ & 1.2 & $1.1-1.2$ & 1.1 & $1.0-1.1$ \\
\hline 70-79 & 1.1 & $1.1-1.2$ & 1.5 & $1.4-1.6$ & 1.2 & $1.1-1.3$ \\
\hline $80+$ & 1.3 & $1.2-1.5$ & 2.3 & $2.0-2.5$ & 1.5 & $1.3-1.7$ \\
\hline \multicolumn{7}{|l|}{ Sex } \\
\hline Male & 1.0 & Ref. & 1.0 & Ref. & 1.0 & Ref. \\
\hline Female & 2.2 & $2.1-2.3$ & 0.8 & $0.8-0.8$ & 2.2 & $2.1-2.3$ \\
\hline
\end{tabular}




\begin{tabular}{|c|c|c|c|c|}
\hline & $\begin{array}{l}\text { No of cases of } \\
\text { distal }^{*} \text { CRC }\end{array}$ & $\begin{array}{l}\text { Distal* }{ }^{*} \text { CRC } \\
\text { incidence }\end{array}$ & Rate ratio $\ddagger$ & $95 \% \mathrm{Cl}$ \\
\hline \multicolumn{5}{|c|}{ Depth of insertion $(\mathrm{cm})$} \\
\hline$<30$ & 3 & 33.2 & 2.9 & $0.9-9.9$ \\
\hline $30-39$ & 7 & 33.8 & 3.0 & $1.2-7.2$ \\
\hline $40-49$ & 5 & 15.5 & 1.4 & $0.5-3.8$ \\
\hline $50-59$ & 9 & 19.9 & 1.9 & $0.8-4.1$ \\
\hline $60+$ & 21 & 11.0 & 1.0 & Ref. \\
\hline \multicolumn{5}{|c|}{ Examination limited by } \\
\hline Spasm/pain & 12 & 22.7 & 1.7 & $0.9-3.4$ \\
\hline Stool & 4 & 9.5 & 0.5 & $0.2-1.5$ \\
\hline Angulation & 10 & 23.5 & 1.7 & $0.8-3.5$ \\
\hline \multicolumn{5}{|c|}{$\begin{array}{l}\mathrm{Cl} \text {, confidence interval. } \\
{ }^{*} \text { Defined as rectum or sigmoid colon. } \\
\dagger \text { Per } 100000 \text { person years. } \\
\ddagger \text { Adjusted for sex, age, and family history of CRC. }\end{array}$} \\
\hline
\end{tabular}

$1.5 \%, 1.4 \%, 1.7 \%$, and $1.7 \%$. These patterns persisted after adjustment for age, sex, and family history of CRC using Poisson regression (data not shown).

By the end of follow up, 45 cases of distal CRC had been diagnosed ( 15 rectal, seven rectosigmoid junction, 23 sigmoid colon). Although prior studies have considered examinations to less than $50 \mathrm{~cm}$ as inadequate, ${ }^{5-7}$ we found that the risk of distal CRC for those with examinations to $40-49 \mathrm{~cm}$ was similar to that in those with examinations to $50-59 \mathrm{~cm}$, and considerably lower than the risk in those with examinations to less than $40 \mathrm{~cm}$ (table 4). For this reason, we have chosen to define "inadequate" examinations as those reaching a depth of less than $40 \mathrm{~cm}$. An indication that the examination was limited by pain, stool, or angulation was not predictive of a further increased risk of distal CRC, once depth of insertion was included in the model. These risks, which were adjusted for age, sex, and family history of CRC, remained essentially unchanged after further adjustment for baseline histological findings, although there was some suggestion that the presence of polyps at baseline was independently associated with subsequent distal CRC diagnosis (RR 1.7 (95\% CI 0.74.1) for non-adenomatous polyps, RR 2.1 (95\% CI 0.9-5.1) for non-advanced adenomas, RR 1.3 (95\% CI $0.2-9.3)$ for advanced adenomas).

\section{DISCUSSION}

Female sex and increasing age were strong risk factors for inadequate sigmoidoscopy, as was unknown provider type and, to a lesser extent, examinations performed by nongastroenterologist physicians. An increase in the number of examinations limited by pain, stool, or angulation only partially accounted for these increased risks; models that either adjusted for limitations or were restricted to those with no limitations showed some attenuations in the risk estimates, but age, sex, and unknown provider type remained strongly associated with inadequate sigmoidoscopy. There was an increased risk of distal CRC among those who had a sigmoidoscopic examination that reached a depth of insertion of less than $40 \mathrm{~cm}$. The presence of limitations of the examination, including spasm/pain, stool, and angulation, did not predict future distal CRC, apart from their association with depth of insertion.

This study has several strengths, most notably the large sample size and availability of follow up data to allow us to quantitate the subsequent risk of distal CRC post-sigmoidoscopy. However, there were several limitations that should be mentioned. Firstly, depth of insertion is only a crude measure of the amount of the colorectum that has been examined. Even among patients with examinations that reach similar depths, the anatomical segment of the colon that is visualised varies. Prior studies have estimated that about $50-75 \%$ of sigmoidoscopies view the entire sigmoid colon, while only a minority of examinations (10-40\%) visualise the entire descending colon. ${ }^{13-15}$ Furthermore, when the CoCaP programme began in 1994, endoscopists were instructed to measure insertion depth based on a straightened sigmoidoscope, with all loops removed. However, no specific quality control measures were instituted to ensure that endoscopists were adhering to this protocol, so it is not known to what degree endoscopists recorded depth of insertion while loops remained. Thus a number of examinations coded as "adequate" may truly have been inadequate according to the actual amount of colon visualised. The net effect of this misclassification however would likely have been to weaken the strength of our observed association, rather than to strengthen it.

Secondly, the possibility of additional misclassification exists in the data, especially because the data were primarily collected for clinical rather than research purposes by multiple endoscopists. For example, underreporting may have occurred in the collection of data regarding limitations of the examination if clinicians failed to note this on the sigmoidoscopy report. Nevertheless, a standardised form was used for all sigmoidoscopies, with items such as limitations in "checkbox" format, to allow clinicians to record data in a consistent and efficient way.

Thirdly, some sigmoidoscopies may have been terminated at the time when a polyp that was not suitable for immediate removal was discovered; these patients would likely have been referred for colonoscopy, making continuation of the sigmoidoscopy unnecessary. Our data support this assertion. Although the prevalence of polyps tended to be greater with increasing depth of sigmoidoscope insertion, those with examinations to less than $30 \mathrm{~cm}$ actually had a higher prevalence of polyps (and especially of advanced adenomas). Thus it seems likely that some small fraction of these shorter examinations was terminated prior to the maximal possible depth of insertion. We attempted to reduce this problem by excluding those sigmoidoscopies that resulted in the diagnosis of cancer. Furthermore, this problem did not result in a large bias; the risk of inadequate examination according to age and sex was almost identical if we restricted to those study subjects who had no polyps identified by sigmoidoscopy, and adjustment for the baseline findings had no impact on the rate ratios for distal CRC according to sigmoidoscopy depth.

Finally, the analyses that examined the risk of distal CRC according to depth of insertion were based on a relatively small number of cases $(n=45)$. Thus our estimate of $40 \mathrm{~cm}$ as the most appropriate cut point in defining "inadequate" 
examinations may have been affected by the limited precision of our risk estimates. Our data are consistent with more modest elevations in cancer risk in those with examinations reaching between 40 and $59 \mathrm{~cm}$ insertion. None the less, this study is, to our knowledge, the first to attempt to quantify the risk of subsequent cancer according to depth of endoscopic insertion.

Our results are largely consistent with those of prior studies examining age $\mathrm{ag}^{5-7}$ and $\operatorname{sex}^{5-9}$ as risk factors for incomplete sigmoidoscopy. In another recent analysis from the Clinical Outcomes Research Initiative (CORI), Walter et al used data from over 15000 examinations in asymptomatic persons performed in multiple sites throughout the USA to assess the risk of inadequate sigmoidoscopy (less than $50 \mathrm{~cm}$ ) by age and sex..$^{5}$ In this population, the proportion of inadequate examinations ranged from $10 \%$ in men aged $50-59$ years to $32 \%$ in women aged 80 years and older. Other recent analyses have had similar findings, ${ }^{67}$ although several other small studies failed to find an association with age ${ }^{16}$ and/or sex. ${ }^{16}$ These results for sigmoidoscopy are also similar to those of several studies that found that older age and female sex were predictive of incomplete or difficult colonoscopy. ${ }^{17-20}$

Our results are also consistent with a those of Schoen and colleagues $^{21}$ who found an increased risk of subsequent advanced colorectal pathology following an inadequate baseline sigmoidoscopy, defined as an examination to less than $50 \mathrm{~cm}$ and/or an examination in which less than $90 \%$ of the colorectal mucosa was visualised due to inadequate preparation (odds ratio 2.7 (95\% CI 1.4-5.4)). This study, conducted among participants in the ongoing PLCO screening trial in the USA, ${ }^{22}$ was based on 72 advanced adenomas; six cases of invasive cancer were also included.

What then accounts for this decreased depth of insertion of the sigmoidoscope in women and older individuals? As suggested in our data, pain, suboptimal bowel preparation, and angulation of the colon account for some, but not all, of these associations. There is other evidence to support these findings; women are more likely to have tortuous colons ${ }^{17} 23$ and to experience pain during sigmoidoscopy, ${ }^{24}{ }^{25}$ while older individuals are more likely to have suboptimal bowel preparation. ${ }^{5826}$ However, other variables are also clearly important predictors. Previous pelvic or abdominal surgery, ${ }^{6-9}$ patient comorbidities, ${ }^{5}$ experience of the endoscopist, ${ }^{927}$ and body weight ${ }^{7}$ have all been reported to be associated with inadequate sigmoidoscopies. We were unable to directly assess these variables with the possible exception of endoscopist experience. Unknown provider type may have provided an estimate of limited endoscopist experience, as those with a lower procedure volume may be more likely to fail to enter a provider identification number on the sigmoidoscopy form or to enter an untraceable number. As certain potentially relevant variables were unavailable to us, it remains possible that at least part of the observed association with age and sex is due to uncontrolled confounding. However, other studies that have controlled for the variables above still found associations with age and sex, ${ }^{5-8}$ although admittedly no study has controlled for all of the above variables. Additionally, it is possible that underreporting of examination limitations by clinicians (as discussed above) could lead to residual confounding when this variable is included in the multivariate models, resulting in risk estimates that are too high.

Despite this, the large magnitude of the associations observed, the precision with which we were able to estimate these associations, and the agreement with prior studies provides strong support for an association of age and sex with inadequate screening flexible sigmoidoscopy. Clinicians should be aware of the potential for limited examinations in older and female individuals, and should consider steps to maximise the potential for an adequate screening examination. For example, several small randomised controlled trials have reported reductions in pain during sigmoidoscopy associated with the use of smaller diameter endoscopes in women, ${ }^{28}$ or with the use of medications ${ }^{29}{ }^{30}$ or audiovisual stimulation $^{31}$ to minimise pain and/or anxiety; in the case of examinations limited by stool, an alternate preparation strategy, such as oral sodium phosphate, could be used. ${ }^{32}$ Alternatively, if an adequate sigmoidoscopy proves difficult, other screening tests can be considered. Because spasm/pain, angulation, and stool cannot fully explain the association between age/sex and inadequate sigmoidoscopy, additional research is needed to better characterise the reasons for inadequate examinations. However, regardless of the reasons, a sigmoidoscopy examination to less than $40 \mathrm{~cm}$ is a cause for concern, due to the increased risk of subsequent CRC.

\section{ACKNOWLEDGEMENT}

This work was supported by the Kaiser Foundation Health Plan Direct Community Benefit Investment Program, and by grants R03CA92767 and 5T32CA09168 from the National Institutes of Health.

\section{Authors' affiliations}

V P Doria-Rose, Division of Public Health Sciences, Cancer Prevention Program, Fred Hutchinson Cancer Research Center, Seattle, WA, USA P A Newcomb, Division of Public Health Sciences, Cancer Prevention Program, Fred Hutchinson Cancer Research Center, Seattle, WA, USA, and Department of Epidemiology, University of Washington, Seattle, WA, USA

T R Levin, Division of Research, Kaiser Permanente Medical Care Program, Oakland, CA, USA

Conflict of interest: None declared.

\section{REFERENCES}

1 Thiis-Evensen E, Hoff GS, Sauar J, et al. Population-based surveillance by colonoscopy: effect on the incidence of colorectal cancer. Telemark Polyp Study I. Scand J Gastroenterol 1999;34:414-20.

2 Newcomb PA, Storer BE, Morimoto LM, et al. Long-term efficacy of sigmoidoscopy in the reduction of colorectal cancer incidence. J Natl Cancer Inst 2003;95:622-5

3 Selby JV, Friedman GD, Quesenberry CPJ, et al. A case-control study of screening sigmoidoscopy and mortality from colorectal cancer. N Engl J Med 1992;326:653-7.

4 Newcomb PA, Norfleet RG, Storer BE, et al. Screening sigmoidoscopy and colorectal cancer mortality. J Natl Cancer Inst 1992;84:1572-5.

5 Walter LC, de Garmo P, Covinsky KE. Association of older age and female sex with inadequate reach of screening flexible sigmoidoscopy. Am J Med 2004; 116:174-8.

6 Eloubeidi MA, Wallace MB, Desmond R, et al. Female gender and other factors predictive of a limited screening flexible sigmoidoscopy examination for colorectal cancer. Am J Gastroenterol 2003:98:1634-9.

7 Ramakrishnan K, Scheid DC. Predictors of incomplete flexible sigmoidoscopy. J Am Board Fam Pract 2003;16:478-84.

8 Stewart BT, Keck JO, Duncan AV, et al. Difficult or incomplete flexible sigmoidoscopy: implications for a screening programme. Aust N Z J Surg 1999;69:19-21.

9 Brill JR, Baumgardner DJ. Establishing proficiency in flexible sigmoidoscopy in a family practice residency program. Fam Med 1997;29:580-3.

10 Palitz AM, Selby JV, Grossman S, et al. The Colon Cancer Prevention Program (CoCaP): rationale, implementation, and preliminary results. HMO Pract 1997;1 1:5-12.

11 Doria-Rose VP, Levin TR, Selby JV, et al. The incidence of colorectal cancer following a negative screening sigmoidoscopy: implications for screening interval. Gastroenterology 2004; 127:714-22.

12 Greenland S. Model-based estimation of relative risks and other epidemiologic measures in studies of common outcomes and in case-control studies. Am J Epidemiol 2004; 160:301-5.

13 Lehman GA, Buchner DM, Lappas JC. Anatomical extent of fiberoptic sigmoidoscopy. Gastroenterology 1983;84:803-8.

14 Jensen J, Kewenter J, Swedenborg J. The anatomic range of examination by fibreoptic rectosigmoidoscopy (60 centimetres). Scand J Gastroenterol 1992;27:842-4.

15 Painter J, Saunders DB, Bell GD, et al. Depth of insertion at flexible sigmoidoscopy: implications for colorectal cancer screening and instrument design. Endoscopy 1999;31:227-31. 
16 Holman JR, Marshall RC, Jordan B, et al. Technical competency in flexible sigmoidoscopy. J Am Board Fam Pract 2001;14:424-9.

17 Church JM. Complete colonoscopy: how often? And if not, why not? Am J Gastroenterol 1994;89:556-60.

18 Cirocco WC, Rusin LC. Factors that predict incomplete colonoscopy. Dis Colon Rectum 1995:38:964-8.

19 Ristikankare M, Hartikainen J, Heikkinen M, et al. The effects of gender and age on the colonoscopic examination. J Clin Gastroenterol 2001;32:69-75.

20 Anderson JC, Messina CR, Cohn W, et al. Factors predictive of difficult colonoscopy. Gastrointest Endosc 2001;54:558-62.

21 Schoen RE, Pinsky PF, Weissfeld JL, et al. Results of a repeat sigmoidoscopy 3 years after a negative examination. JAMA 2003;290:41-8

22 Kramer BS, Gohagan J, Prorok PC, et al. A National Cancer Institute sponsored screening trial for prostatic, lung, colorectal, and ovarian cancers. Cancer 1993;71:589-93.

23 Saunders BP, Fukumoto $M$, Halligan $S$, et al. Why is colonoscopy more difficult in women? Gastrointest Endosc 1996;43:124-6.

24 UK Flexible Sigmoidoscopy Screening Trial Investigators. Single flexible sigmoidoscopy screening to prevent colorectal cancer: baseline findings of a UK multicentre randomised trial. Lancet 2002;359:1291-300.
25 Schoen RE, Weissfeld JL, Bowen NJ, et al. Patient satisfaction with screening flexible sigmoidoscopy. Arch Intern Med 2000;160:1790-6.

26 Ness RM, Manam R, Hoen H, et al. Predictors of inadequate bowel preparation for colonoscopy. Am J Gastroenterol 2001;96:1797-802

27 Hawes R, Lehman GA, Hast J, et al. Training resident physicians in fiberoptic sigmoidoscopy. How many supervised examinations are required to achieve competence? Am J Med 1986;80:465-70.

28 Farraye FA, Horton K, Hersey H, et al. Screening flexible sigmoidoscopy using an upper endoscope is better tolerated by women. Am J Gastroenterol 2004:99:1074-80.

29 Harding TA, Gibson JA. The use of inhaled nitrous oxide for flexible sigmoidoscopy: a placebo-controlled trial. Endoscopy 2000;32:457-60.

30 Kuganeswaran E, Clarkston WK, Cuddy PG, et al. A double-blind placebo controlled trial of oral midazolam as premedication before flexible sigmoidoscopy. Am J Gastroenterol 1999:94:3215-19.

31 Lembo T, Fitzgerald L, Matin K, et al. Audio and visual stimulation reduces patient discomfort during screening flexible sigmoidoscopy. Am J Gastroenterol 1998;93:1113-16.

32 Bini EJ, Unger JS, Rieber JM, et al. Prospective, randomized, single-blind comparison of two preparations for screening flexible sigmoidoscopy Gastrointest Endosc 2000;52:218-22.

\section{EDITOR'S QUIZ: GI SNAPSHOTS}

\section{A challenging presentation of left upper quadrant pain}

\section{Clinical presentation}

A 21 year old female was admitted with a 48 hour history of severe left upper quadrant and loin pain with radiation to the shoulder tip, nausea, and vomiting. She was para $1+0$ with a past medical history of a negative laparoscopy for investigation of mennorhagia seven weeks prior to admission. Physical examination revealed a pyrexia of $38.4^{\circ} \mathrm{C}$ and sinus tachycardia of 120 beats per minute. She had severe tenderness and guarding in the left upper quadrant and loin, in addition to left sided basal crepitations. Blood tests revealed a white cell count of $23 \times 10^{9} / \mathrm{l}$, platelet count of $312 \times 10^{9} / \mathrm{l}$, and $\mathrm{C}$ reactive protein of $300 \mathrm{mg} / \mathrm{l}$. The remainder of her blood investigations were within the normal range.

Chest $x$ ray revealed left basal consolidation. Abdominal ultrasound scan showed a left sided subdiaphragmatic collection associated with other abnormalities which were investigated further by subsequent computed tomography scan (fig 1).

\section{Question}

What are the abnormalities noted at A and B in fig 1 ? What is the diagnosis and management?

See page 1317 for answer

This case is submitted by:

R Taghizadeh, B K Chew

Canniesburn Plastic Surgery Unit, Glasgow Royal Infirmary, Glasgow, UK

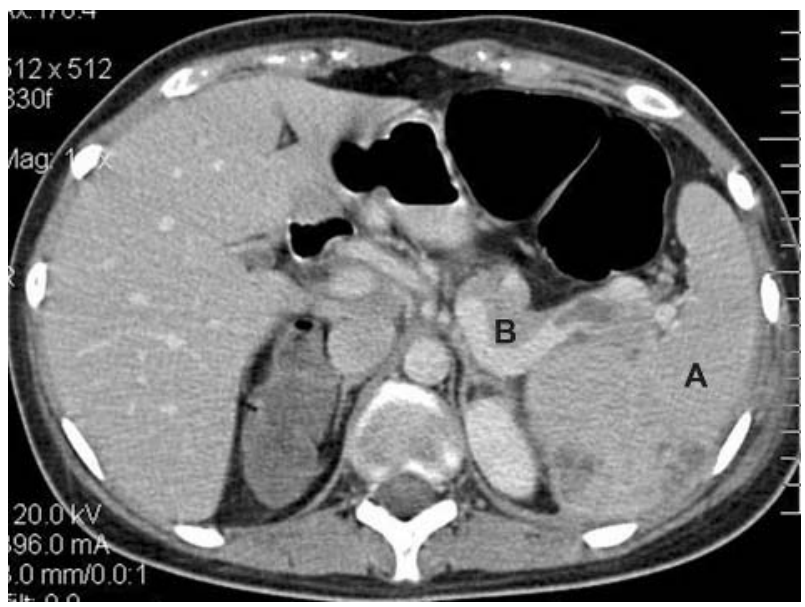

Figure 1 Splenomegaly $(\mathrm{A})$ and extensive thrombus within the splenic vein (B).

K G Mitchell

Department of General Surgery, Royal Alexandra Hospital, Paisley, UK

Correspondence to: Dr R Taghizadeh, Canniesburn Plastic Surgery Unit, Glasgow Royal Infirmary, Glasgow G61 1NB, UK; rieka78taghizadeh@yahoo.com

doi: 10.1136/gut.2004.059063 